\title{
Education Workshop
}

\section{E-1}

Education Kit for Determining Cell Viability, Death and Apoptosis from the Same Sample. TERRY L. RISS. Promega, 2800 Woods Hollow Road, Madison, WI 537110-5399. Email: terry.riss@promega.com

The ApoTox-Glo ${ }^{\text {TM }}$ Triplex Assay combines three assay chemistries to assess viability, cytotoxicity and caspase activation events within a single assay well. The first part of the assay simultaneously measures two protease activities; one is a marker of cell viability, and the other is a marker of cytotoxicity. The live-cell protease activity is restricted to intact viable cells and is measured using a fluorogenic, cell-permeable, peptide substrate (glycylphenylalanyl-aminofluorocoumarin; GF-AFC). The substrate enters intact cells where it is cleaved by the live-cell protease activity to release AFC and generate a fluorescent signal proportional to the number of living cells. This live-cell protease becomes inactive upon loss of cell membrane integrity and leakage into the surrounding culture medium. A second, fluorogenic cell-impermeant peptide substrate (bis-alanylalanyl-phenylalanyl-rhodamine 110; bis-AAF$\mathrm{R} 110$ ) is used to measure dead-cell protease activity, which is released from cells that have lost membrane integrity. Because bis-AAF-R110 is not cell-permeable, essentially no signal from this substrate is generated by intact, viable cells. The live- and dead-cell proteases produce different products, AFC and R110, which have different excitation and emission spectra, allowing them to be detected simultaneously. The second part of the assay uses the Caspase-Glo ${ }^{\circledR}$ Assay to provide a luminogenic caspase-3/7 substrate, which contains the DEVD amino acid sequence in a reagent optimized to measure caspase activity. Adding the Caspase-Glo ${ }^{\circledR}$ 3/7 Reagent in an "add-mix-measure" format results in cell lysis, followed by caspase cleavage of the substrate and generation of a "glow-type" luminescent signal produced by luciferase. Luminescence is proportional to the amount of caspase activity present. The Caspase-Glo ${ }^{\circledR}$ 3/7 Reagent relies on the properties of a proprietary thermostable luciferase (Ultra-Glo ${ }^{\mathrm{TM}}$ Recombinant Luciferase), which is formulated to generate a stable "glow-type" luminescent signal and improve performance across a wide range of assay conditions. 